\title{
Philosophiques
}

\section{La fondation de la bioéthique : une perspective épistémologique}

\section{François Tournier}

Volume 16, numéro 2, automne 1989

URI : https://id.erudit.org/iderudit/027081ar

DOI : https://doi.org/10.7202/027081ar

Aller au sommaire du numéro

Éditeur(s)

Société de philosophie du Québec

ISSN

0316-2923 (imprimé)

1492-1391 (numérique)

Découvrir la revue

Citer cet article

Tournier, F. (1989). La fondation de la bioéthique : une perspective épistémologique. Philosophiques, 16(2), 257-291.

https://doi.org/10.7202/027081ar
Résumé de l'article

L'entreprise fondationnelle traditionnelle en éthique a abouti à un échec, c'est-à-dire à la victoire du relativisme moral. De son côté, la métaéthique a contribué largement à renforcer ce relativisme en voulant caractériser l'éthique négativement en prenant pour point de référence le discours scientifique (ou les énoncés descriptifs). Mais n'y aurait-il pas une autre façon de fonder l'éthique et la bioéthique ? Le présent texte voudrait apporter réponse à cette question en adoptant une perspective épistémologique. 


\title{
LA FONDATION DE LA BIOÉTHIQUE : UNE PERSPECTIVE ÉPISTÉMOLOGIQUE
}

\author{
par François Tournier
}

\begin{abstract}
RÉSUMÉ. L'entreprise fondationnelle traditionnelle en éthique a abouti à un échec, c'est-à-dire à la victoire du relativisme moral. De son côté, la métaéthique a contribué largement à renforcer ce relativisme en voulant caractériser l'éthique négativement en prenant pour point de référence le discours scientifique (ou les énoncés descriptifs). Mais n'y aurait-il pas une autre façon de fonder l'éthique et la bioéthique? Le présent texte voudrait apporter réponse à cette question en adoptant une perspective épistémologique.
\end{abstract}

ABSTRACT. In our view, the field of ethics is in jeopardy: the traditional approaches wanting to give an ontological foundation to ethics has lead on the contrary (by it's constant failure to find them) to the victory of moral relativism; on the other hand, further support has been provided for relativism with the intervention of metaethics which defines ethics negatively in contrast to science (or descriptive sentences). Could there be another way to secure the foundation of ethics? In this paper, we try to see whether the perspective of the philosophy of science could not help us to choose rationally between moral rules and in this way to provide an indirect foundation for ethics and bioethics but nevertheless a foundation.

Le titre de cet exposé mentionne le nom de deux disciplines dont la signification et la référence demeurent assez vagues du fait de leurs présentations différentes d'un auteur à l'autre; aussi importe-t-il de commencer notre analyse en précisant ce que nous entendrons par les expressions «épistémologie » et «bioéthique ». Notons, au départ, la relation théorique que nous établissor,s entre les deux : la bioéthique constitue l'objet de la présente recherche et nous emprunterons à l'épistémologie contemporaine sa perspective qui deviendra ainsi notre grille d'analyse. Pour le formuler en termes kantiens, l'épistémologie nous procure les catégories synthétiques a priori (les catégories de l'entendement) nécessaires à la 
caractérisation de notre objet, la bioéthique. À nos yeux, l'intérêt d'une telle analyse est de percevoir la bioéthique avec un regard neuf et ainsi de faire apparaître des enjeux théoriques qui autrement seraient restés dans l'ombre. Nous y soutiendrons bien sûr une thèse mais celle-ci est épistémologique et non pas éthique.

L'épistémologie sera considérée ici comme un discours de second niveau prenant pour objet, non pas le monde, mais le discours scientifique et tentant de montrer que notre croyance en l'authenticité des connaissances humaines qui y sont véhiculées peut être rationnellement justifiée (du moins en principe). Nous disons «montrer» et non pas «démontrer» car, à nos yeux, l'épistémologie n'est pas une science formelle comme les mathématiques ou la logique et elle n'est pas une science expérimentale non plus. Son statut est assez similaire à celui de l'éthique si ce n'est qu'elle cherche à fonder rationnellement non pas des règles morales mais bien des règles méthodologiques. Dans la perspective épistémologique, la bioéthique est un discours, c'est-à-dire un ensemble de propositions (ou d'énoncés) organisées entre elles d'une façon caractéristique. Cette façon de considérer la bioéthique distingue notre perspective de celle de d'autres disciplines comme la sociologie, la psychologie ou l'histoire. Notre entreprise consistera donc à montrer à quelles conditions (c'est-à-dire en respectant quelles règles méthodologiques dans la formulation et l'organisation des propositions éthiques) notre croyance en l'authenticité des connaissances en bioéthique (les règles morales) peut être rationnellement justifiée.

D'emblée deux objections s'imposent : l'éthique traditionnelle jusqu'à nos jours n'a rien fait d'autre que de tenter de justifier rationnellement les règles morales et la métaéthique qui est apparue au début du siècle avec l'ouvrage de G.E. Moore, Principia Ethica (1903), adopte, comme nous entendons le faire, une perspective de second niveau dans son analyse du discours éthique. La perspective épistémologique ne serait donc pas aussi nouvelle qu'on voudrait le prétendre. Contrairement à l'éthique traditionnelle nous ne tenterons pas de fonder des règles morales particulières sur des conceptions de l'être humain ou de l'univers ou sur des principes impératifs et catégoriques. Nous pensons même qu'un constat d'échec total est manifeste en ce qui concerne ce genre d'entreprise qui de toute évidence devrait être abandonnée. En 
fait, ces recherches ont abouti exactement au contraire de ce qu'elles visaient, à savoir, à la victoire du relativisme moral. Mais ce qui nous distingue le plus de ce genre d'approches est que la thèse qui sera défendue ici est épistémologique et non pas éthique. Nous tenterons plutôt d'établir à quelles conditions méthodologiques un discours éthique quelconque sera rationnellement justifiable, c'est-à-dire, préférable à un autre. En ce sens, nous nous situerons au niveau d'une théorie générale de la bioéthique, comme la métaéthique, mais au contraire de celle-ci qui insiste exclusivement sur la différence entre le discours éthique et le discours scientifique, nous mettrons l'accent sur leur ressemblance et leur compatibilité. Enfin, notre recherche diffère d'autres plus récentes comme celles de J.C. Harsanyi $(1982,1976)$ et de David Gauthier (1986) en ce qu'elle ne vise pas à fonder rationnellement des actions mais bien un certain type de discours.

\section{LA STRUCTURE ÉPISTÉMOLOGIQUE DU DISCOURS BIOÉTHIQUE}

Un discours bioéthique comprend un ensemble d'énoncés dont les plus significatifs épistémologiquement sont les suivants : 1. des descriptions de situations particulières ayant des implications morales, c'est-à-dire dans lesquelles interviennent des comportements (des actions ou des inactions) susceptibles d'être approuvés ou blâmés en vertu de certaines règles morales : par exemple, « Au temps t et à l'hôpital $\mathrm{y}$, un médecin $\mathrm{x}$ a décidé de ne pas révéler tout de suite à la patiente $z$ qu'elle était atteinte d'un cancer ne lui laissant que six mois à vivre. "; ce comportement est approuvé par la règle morale nous enjoignant d'être charitable envers son prochain en ne lui révélant pas trop brutalement sa condition médicale, et condamné par une autre règle voulant qu'on respecte l'autonomie d'une personne humaine en lui révélant une information pouvant lui servir à prendre des décisions au sujer de son avenir ; 2. des jugements moraux, c'est-à-dire une évaluation (positive ou négative) de la moralité de certains comportements particuliers dans des situations particulières: par exemple, « le psychologue $\mathrm{x}$ n'a pas agi d'une façon éthiquement correcte en ne révélant pas à la victime avant son meurtre que son patient $z$ qui était l'amant de la victime, lui avait affirmé avant de le quitter qu'il allait la tuer. »; le comportement est approuvé par la règle morale voulant qu'on garde le secret professionnel au sujet de la vie privée des gens et 
condamné par une autre règle voulant qu'on respecte la vie humaine; ici, le jugement moral vient trancher le conflit en accordant priorité au respect de la vie; mais le jugement moral ne fait pas qu'accorder priorité à certaines règles morales car, préalablement, il doit établir que ce comportement particulier dans ces circonstances particulières est un cas particulier d'une ou de plusieurs règles morales; 3 . des descriptions de situations typiques ayant valeur d'exemple: par exemple, «des médecins font de la recherche sur des enfants en bas âge » ou « des médecins pratiquent des interventions chirurgicales dangereuses pour la vie du patient même si les résultats escomptés sont minimes en ce qui regarde l'amélioration de son état de santé »; 4 . des règles morales, c'està-dire des évaluations générales pouvant guider des comportements typiques dans des situations typiques: par exemple, «la recherche médicale sans but thérapeutique pratiquée à l'aide de jeunes enfants devrait être strictement interdite "; 5 . des principes moraux (catégoriques ou hypothétiques, nous reviendrons sur cette distinction plus loin), c'est-à-dire des règles d'un niveau de généralité plus élevé que les règles morales du fait que les principes appartiennent au champ plus général de l'éthique (et non à celui de la bioéthique) et qu' ils servent à justifier les règles morales en montrant que ce ne sont que des cas particuliers d'un principe plus général: par exemple, le principe du consentement volontaire et le principe du respect de l'autonomie de la personne justifient la règle morale interdisant la recherche sans but thérapeutique sur les jeunes enfants et peuvent justifier tout autant une loi dans un tout autre domaine interdisant la publicité pour les enfants; 6. des valeurs suprêmes comme la personne humaine dans la philosophie éthique de Kant ou le bonheur dans celle d'Aristote dont la fonction est d'introduire une hiérarchie permettant d'ordonner par ordre de priorité les principes moraux pouvant, comme nous l'avons vu au sujet des règles morales, entrer en conflit; 7 . des conceptions générales extra-morales comme des conceptions de l'être humain, de la rationalité, de la société, de l'univers, etc.

Les différents éléments du discours bioéthique ainsi posés, considérons un concept théorique introduit par les épistémologues contemporains (comme C.G. Hempel, 1972:85-6 ou K.R. Popper, 1959: 62-4; 68-70), à savoir, celui de niveau d'universalité, qui nous servira à les organiser de façon compréhensible. Selon ces 
conceptions épistémologiques, un énoncé existentiel singulier (un énoncé affirmant l'existence d'un fait) comme «À l'hôpital SaintLuc de Montréal, le 30 janvier à 15:30 hrs, le docteur Lucienne Grandcour a arrêté l'appareil maintenant artificiellement en vie la patiente Caroline Ledoux et celle-ci est morte. », est d'un niveau d'universalité ou de généralité inférieur à un énoncé ayant la forme d'un énoncé strictement universel comme «Toutes les fois que le fonctionnement d'un appareil maintenant artificiellement en vie une personne cesse, celle-ci meurt.». En fait, si cet énoncé a la forme d'un énoncé strictement universel, ce n'en est pas un. En plus d'avoir une forme universelle un énoncé strictement universel n'admet pas d'exception, c'est-à-dire qu'il y a une connexion nécessaire entre les termes de l'énoncé. Or, il est clair qu'il n'y a pas de connexion nécessaire entre le fonctionnement de certains appareils et la mort d'une personne: ce n'est qu'en certaines occasions très spéciales où les deux seront liés par un ensemble d'éléments intermédiaires (comme le type d'appareil impliqué, la condition médicale de la patiente, etc.). Un énoncé strictement universel authentique serait "Toutes les fois que le cœur d'une personne (non maintenue artificiellement en vie) cesse de battre pendant un temps $t$ alors cetre personne meurt " car nous savons qu'il y a une connexion nécessaire naturelle (sans exception) entre le battement du cœur et le maintien des fonctions vitales. Le faux énoncé universel mentionné plus haut est en fait une généralisation accidentelle, c'est-à-dire qu' elle est vraie dans certains cas et fausse dans d'autres. Nous surnommerons ce dernier type d'énoncé des énoncés universels singularisés car ils ne sont vrais que dans quelques cas particuliers comme «Toutes les auditrices ici présentes comprennent le français » ou «Toutes les praticiennes de la santé dans cet hôpital sont en faveur de l'avortement libre ». Le dernier type d'énoncé que nous voudrions introduire est l'énoncé existentiel généralisé qui s'obtient en faisant abstraction des coordonnées spatio-temporelles et des noms propres dans un énoncé existentiel singulier : pour reprendre l'exemple ci-haut, «Il existe au moins un cas où une femme médecin a fait cesser le fonctionnement de l'appareil maintenant artificiellement en vie une personne.» Ces distinctions posées, faisons abstraction de ce qu'il y a de spécifiquement moral dans le discours bioéthique et dressons un tableau combinant les catégories épistémologiques présentées jusqu'ici. 
Conceptions générales extra-morales

Les valeurs suprêmes (hiérarchie de valeurs)

Les principes moraux catégoriques

Les principes moraux hypothétiques

Les règles morales

Des descriptions typiques

(de comportements typiques dans des circonstances typiques)

Les jugements moraux

Les descriptions singulières (de comportements particuliers dans des circonstances particulières)
Tableau 1

ex. : L'être humain est rationnel ou égoïste

Énoncé strictement universel

ex. : Le respect de la personne

Énoncé strictement universel

ex. : Toute personne doit être traitée comme une fin en soi

ex. : On doit respecter la liberté d'agir de toute personne à moins que notre propre sécurité soit menacée

ex. : La recherche médicale sans but thérapeutique sur de jeunes enfants est éthiquement condamnable

ex. : La recherche médicale sur de jeunes enfants est pratiquée

ex. : Le comportement $\mathrm{x}$ de la chirurgienne y dans les circonstances $c$ est éthiquement condamnable

ex. : Dans les

circonstances c, la chirurgienne $\mathrm{x}$ a

Énoncé strictement universel

Énoncé universel singularisé

Énoncé existentiel généralisé

Énoncé existentiel généralisé

Énoncé existentiel singulier pratiqué l'intervention chirurgicale z
Énoncé existentiel singulier

Dans ce tableau, la seule distinction n'ayant pas fait l'objet d'explicitations antérieures est celle entre les principes moraux catégoriques et hypothétiques. Dans l'esprit de Kant qui introduit une distinction entre des « impératifs catégoriques » (inconditionnels à aucune fin) et des «impératifs hypothétiques » (conditionnels à certaines fins), nous voudrions établir une distinction similaire au niveau des principes moraux. Notre distinction diffère de celle de Kant cependant en ce que nous insistons uniquement sur l'aspect inconditionnel (au sens d'universel et sans exception) et sur 
l'aspect conditionnel (au sens d'exceptionnellement universel et comportant des exceptions) de ces deux types de principes moraux. Contrairement à Kant, le fair que certains impératifs dépendent de fins contingentes alors que d'autres dépendent de principes absolus, ne retiendra pas notre attention ici. D'ailleurs, à notre avis, Perelman (1980: 127) a raison de voir dans cette croyance en des absolus comme le signe de l'utopisme (le caractère inapplicable, dirons-nous) de l'éthique kantienne :

Elle est sans doute utopique lorsqu'elle nie l'existence possible de conflits de devoirs : lorsque deux devoirs semblent s'opposer, dit-elle, il y a apparence de conflit car en réalité, des deux devoirs, l'une emporte sur l'autre.

Contrairement à Kant donc, notre distinction n'a pas pour but d'affirmer qu'il existe des impératifs catégoriques mais bien de départager entre des principes moraux qui sont inconditionnels, universels et sans exception, et d'autres qui sont conditionnels, singularisés et comportant des exceptions. Les premiers n'admettent aucune transgression, ont toujours priorité sur les principes hypothétiques et s'appliquent mécaniquement. Les seconds admettent quelquefois d'être transgressés, la question de leur priorité doit être débattue et leur application est le fruit d'une réflexion pesant le pour et le contre. Cette distinction veut donc insister sur le fait que des principes moraux peuvent entrer en conflit avec d'autres. Certes «Il ne faut pas tuer son prochain" mais lorsqu'il s'agit de défendre sa propre existence ou de brûler des hérétiques ou des sorcières, il faut bien faire une exception à ce principe moral en apparence absolu. La question, non pas de l'existence, mais de la justification rationnelle possible de principes moraux absolus en éthique et en bioéthique sera débattue plus loin.

Le tableau 1 met en correspondance les niveaux d'universalité des énoncés avec leur caractéristique épistémologique d'être des principes, des règles ou des jugements moraux. Au niveau d'universalité le plus bas (celui de l'énoncé existentiel singulier), nous faisons correspondre la description de situations particulières et les jugements moraux. À un niveau de généralité un peu plus élevé (celui de l'énoncé existentiel généralisé), nous faisons correspondre la description de situations typiques et les règles morales. À un niveau supérieur (celui des énoncés universels singularisés), nous faisons correspondre les principes moraux 
hypothétiques. Enfin, les autres composantes du discours bioéthique (les principes moraux catégoriques, les valeurs suprêmes et les conceptions générales extra-morales) se situent au niveau le plus élevé, celui des énoncés strictement universels.

\section{LE STATUT LOGIQUE DES ÉNONCÉS BIOÉTHIQUES}

Maintenant que nous avons caractérisé épistémologiquement le discours bioéthique, considérons le statut logique de ces énoncés qui, comme nous le savons, sont de différents niveaux d'universalité. Pour ce faire, introduisons une autre catégorisation épistémologique qui remonte au débat entre Kant et Hume et qui a fait, ces dernières années, l'objet de critiques sérieuses : notamment, celles de Quine (1953) et de Kripke (1982). Il s'agit de la distinction entre analy tique et synthétique. Mais comme nous entendrons par «analytique» une vérité logique (une tautologie) et que nous ne confondrons pas les sens épistémique et ontologique de ces termes, nous nous croyons tout à fait justifiés de l'utiliser pour faire apparaître une caractéristique importante du discours bioéthique. Les catégories «analytique " et «synthétique» seront utilisées ici non pas pour caractériser la nature (syntaxique ou sémantique) d'un énoncé mais bien l'usage qu'on entend faire de cet énoncé. On ne saurait considérer les énoncés de la bioéthique (ou de l'éthique) comme des énoncés synthétiques au même titre que les énoncés des sciences expérimentales sans les rendre complètement inaptes à remplir leur fonction spécifique. La différence entre «loi de la nature» et «règle» (énoncé descriptif et énoncé normatif) est suffisamment connue pour avoir nul besoin d'insister sur ce point. Par exemple, si une règle de la circulation (comme l'interdiction de traverser au feu rouge) était assimilée à un énoncé synthétique, il nous faudrait la déclarer invalide et la rejeter à chaque fois qu'un agent de la paix émet une contravention à cet effet. Contrairement à un énoncé synthétique qu'on déclare invalide lorsqu'il est contredit par des énoncés décrivant des faits empiriques, pour remplir sa fonction, l'énoncé éthique doit rester valide même s'il est constamment contredit par les faits (c'est-à-dire quand la règle est transgressée). On exprime le plus souvent cette différence en disant que les énoncés moraux ne sont pas descriptifs mais prescriptifs ou normatifs. La validité d'un tel énoncé ne peut donc venir de sa correspondance au monde. 
Mais cette différence n'est pas suffisante, à notre avis, pour en conclure que la validité d'un énoncé moral est complètement indépendante de ce qui se passe dans le monde. On ne saurait non plus faire de ce genre d'énoncé des énoncés analytiques, (au sens de vérité logique) comme ceux des mathématiques et de la logique sans également les rendre inaptes à remplir leur fonction. Supposons par exemple qu'un agent de la paix vous arrête parce que vous avez enfreint un règlement municipal et que vous lui demandiez pourquoi il vous a arrêté ? S'il vous répond que tous ceux qui enfreignent la loi sont arrêtés, il aura sûrement fondé son arrestation mais non pas satisfait votre curiosité. Ce que vous voulez plutôt savoir c'est ce que vous avez fait, c'est-à-dire lequel de vos comportements antérieurs enfreint un règlement municipal et de quel règlement il s'agit? Supposons maintenant qu'il vous réponde que vous avez transgressé le règlement du bonheur, vous lui demanderez certainement quel est ce règlement? Supposons qu'il vous réponde que le bonheur est ce que tout le monde veut et donc que si vous êtes arrêté, c'est que vous n'avez pas été conforme à ce que tout le monde veut. Mais que veut le monde, demanderezvous? Tout le monde veut le bonheur rétorquera l'agent de la paix d'un air visiblement agacé par toutes vos questions. En fait, «tout le monde veut le bonheur " et « le bonheur est ce que tout le monde veut » forment conjointement une vérité logique et il importe qu'un discours éthique puisse sortir de cette circularité s'il doit servir de guide dans nos actions. On a traditionnellement caractérisé cet aspect de discours moral en le qualifiant de «savoir pratique». C'est pourquoi nous ne pouvons qu'être d'accord avec Jocelyne Couture (1986: 17) face à la perspective adoptée par la tradition issue de Moore en métaéthique qui consiste largement à caractériser le discours moral par la négative en prenant pour point de référence absolu les énoncés synthétiques des sciences de la nature :

$[\ldots]$ l'analyse du discours moral ne peut plus se satisfaire des approches dites linguistiques produites dans la lignée de Moore et qui, elle aussi, en se concentrant sur une analyse des termes moraux, se sont adonnée au jeu réducteur de la «bivalence». Tout ce qu'une analyse conceptuelle ou logique des termes moraux peut établir au sujet de la spécificité du discours moral, c'est au moins que celui-ci ne possède pas les propriétés logiques du discours constatif et au plus qu'il possède les propriétés générales du discours performatif où on trouve des termes similaires. 
C'est pourquoi Jocelyne Couture recommandera de se tourner vers l'étude du raisonnement pour saisir la spécificité du discours moral en tant que savoir pratique. Pour notre part, nous tenterons de caractériser épistémologiquement cette spécificité en disant que pour pouvoir remplir sa fonction (guider des comportements), le discours moral doit pouvoir se ramener à des énoncés existentiels généralisés décrivant des comportements typiques dans des situations typiques et à des énoncés existentiels singuliers décrivant des comportements particuliers dans des circonstances particulières. S'il en était autrement, le discours moral ne pourrait tout simplement pas guider l'action. Ce type de discours ne comporte pas seulement un aspect normatif mais également un aspect descriptif même si la non-correspondance aux faits empiriques ne peut l'invalider. En ce sens, il représente une combinaison particulière d'énoncés analytiques et synthétiques, de sorte qu'on pourra établir sa validité de façon partiellement a priori et partiellement a posteriori.

Mais le discours moral possède également un autre aspect, ontologique, qui a surtout été développé par la philosophie traditionnelle. Il ne serait certainement pas exagéré de vouloir caractériser, à la suite de Perelman (1980: 201), la philosophie morale traditionnelle remontant à Platon et à Aristote comme une entreprise visant à fonder la morale (les règles et les principes) sur la raison:

En effet, l'objectif de la philosophie morale est de chercher un fondement rationnel à la morale, fondement qui ne soit pas simplement le commandement divin, la tradition sociale ou une quelconque moralité donnée.

La philosophie morale cherche à déduire la morale d'une certaine conception de l'homme ou de ses rapports avec la société, d'une vision de l'univers ou du cosmos.

Jusqu'ici la perspective traditionnelle a donc consisté à se situer au plus haut niveau d'universalité du discours éthique et à tenter d'en déduire logiquement les principes et les règles morales. On voudrait de ce fait leur conférer un fondement rationnel. C'est en ce sens que la philosophie morale traditionnelle est une entreprise de fondation. Tirons ici la conséquence qui s'impose. Si la contradiction entre une règle morale et des faits empiriques n'est pas susceptible de l'invalider, il semble qu'une telle contradiction, 
mais avec des conceptions ontologiques extra-morales, peut le faire. Ainsi, si une règle morale vient contredire notre conception de l'être humain et si nous accordons priorité à notre conception, nous serons portés tout naturellement à invalider la règle morale et à la rejeter. C'est pourquoi jusqu' ici l'entreprise de fondation de l'éthique s'est faite d'une façon purement a priori et que l'impossibilité de s'entendre sur des conceptions extra-morales universelles est la principale cause de l'échec à lui trouver un fondement universel. Il serait certainement plus facile d'en arriver à un consensus sur des indices moins universels et a posteriori. De ce point de vue, la métaéthique n'a rien de très « révolutionnaire» car ses analyses de termes et de raisonnements moraux se font également de façon uniquement a priori. Mais, comme nous venons de le voir, à côté des aspects ontologique et normatif qui jusqu' ici ont servi à justifier et à analyser le discours moral, il y a un aspect descriptif dont il importe d'examiner les implications pour le problème de la validité des énoncés moraux.

\section{L'ASPECT SYNTHÉTIQUE DU DISCOURS MORAL}

En accord avec Perelman (1980 : 204), nous dirons que :

Le grand problème n'est pas celui de leur fondement mais de leur application : quand a-t-on le droit de faire souffrir un être? Qu'est-ce que le bonheur, l'utilité, l'ordre? Voilà les vrais problèmes de la morale: la mise en rapport des règles abstraites avec des situations concrètes.

Cette nouvelle façon d'envisager le problème de la fondation de l'éthique et de la bioéthique implique un double renversement de perspective: 1 . renversement de la perspective traditionnelle qui centrait la recherche au plus haut niveau d'universalité : il faut plutôt, à notre avis, faire porter la recherche sur le plus bas niveau d'universalité (le rapport entre les énoncés normatifs et les énoncés descriptifs) ; 2 . renversement de la perspective métaéthique cherchant à montrer qu'on ne peut déduire un énoncé prescriptif d'un énoncé descriptif: nous nous demanderons plutôt s'il est possible de déduire un énoncé descriptif d'un énoncé normatif ?

Pour s'attaquer à la philosophie morale traditionnelle, la métaéthique tracera une ligne de démarcation nette entre jugement de fait et jugement de valeur. 
Si on devait admettre, écrit Perelman (1980: 201), cette séparation nette entre jugement de fait et jugement de valeur, on montrerait en même temps que toute la philosophie morale traditionnelle est fondée sur une illusion.

En effet, comme nous l'avons mentionné, l'entreprise éthique traditionnelle voulait déduire des énoncés normatifs à partir d'énoncés ontologiques. Déjà, David Hume dans son Traité sur la nature bumaine faisait remarquer qu'on ne pouvait déduire logiquement ce qui doit être à partir de ce qui est. Cette critique sera reprise par Moore (1903) qui surnommera certe erreur des philosophes moraux traditionnels, le « sophisme naturaliste », qui consiste à croire qu'on pourrait déduire des énoncés normatifs de prémisses ne contenant que des énoncés descriptifs. Mais l'erreur n'est pas aussi évidente que ne le supposait Hume puisque le débat persiste toujours même à l'intérieur de la tradition de la philosophie analytique: cf. Hudson (ed.) (1969) et Searle (1969: chap. 8).

Mais, comme nous le disions, une autre combinaison des énoncés normatifs et descriptifs, celle qui consiste à déduire des énoncés descriptifs à partir de prémisses contenant des énoncés normatifs, est possible et c'est celle qui nous intéressera ici. En fait, le sophisme de Hume se voulait une critique de la philosophie morale traditionnelle mais, en inversant l'ordre de la déduction, notre analyse concerne plutôt toute éthique qu'elle soit traditionnelle ou non. En effet, pour pouvoir remplir sa fonction (guider l'action), toute éthique doit contenir des énoncés normatifs pouvant s'appliquer à des actions particulières dans des situations concrètes. C'est là une condition de possibilité de toute éthique sur laquelle insiste J.N. Kaufmann dans le passage suivant (1987a: 11):

Dans la perspective kantienne, l'impératif du devoir stipule de n'accepter comme maxime de l'action que celles qui se conforment à l'impératif catégorique : [...] Mais l'impératif catégorique ne permet pas à lui seul de spécifier de quoi je suis obligé, à moins que l'on accepte un autre principe stipulant que ce que l'impératif catégorique ne défend pas est obligatoire (...) Bien que ce principe additionnel autoriserait à tirer de l'impératif catégorique des devoirs possibles, il ne permettrait pas, néanmoins d'indiquer quels devoirs déterminés incombent à des agents particuliers dans des circonstances déterminées. La question est de savoir comment des expressions « avoir le devoir de faire », "être sous l'obligation de $»$, etc. s'appliquent. 
En bref, le problème de la relation entre les énoncés normatifs et descriptifs au plus bas niveau d'universalité du discours moral nous apparaît plus fondamental que celui consistant à déduire des énoncés normatifs d'énoncés ontologiques (la perspective de la philosophie morale traditionnelle) ou que celui qui consiste à montrer qu'une telle déduction est possible ou non (la perspective métaéthique). Logiquement, quand bien même on réussirait à déduire et à ordonner hiérarchiquement les règles et les principes moraux à partir d'une ontologie, ou même qu'on réussirait à démontrer formellement qu'une telle déduction est impossible, la question de l'application d'une éthique quelconque à des comportements particuliers dans des conditions particulières demeure entièrement ouverte. Il y va de la possibilité même d'une éthique qui se veut non seulement normative (des règles morales rationnellement fondées) mais surtout applicable à des situations concrètes. Mais comme l'éthique s'est toujours voulue un savoir pratique et non un savoir analytique (la déduction pure d'énoncés à partir d'autres énoncés plus fondamentaux), nous nous croyons entièrement justifiés de vouloir changer la perspective sur ces questions et insister d'avantage sur «l'aspect technologique » de tout discours éthique.

Dans le cas spécifique de la bioéthique, nous pouvons ajouter à cet argument logique, un autre argument historique. $\grave{A}$ notre avis, l'intérêt d'une entreprise visant à fonder rationnellement la bioéthique devrait être entièrement centré sur les niveaux les plus bas d'universalité de son discours non seulement parce que la question est logiquement plus fondamentale mais également parce que historiquement cette discipline est née à partir de problèmes concrets posés par les développements de la médecine et de la biologie. La bioéthique n'est pas une conséquence logique tirée d'un système moral élaboré par un philosophe. Les problèmes moraux de la bioéthique, écrit T.L. Beauchamp (1982: 1) «ont surgi de la pratique professionnelle dans les champs de la médecine clinique, de la recherche biomédicale, chez le personnel infirmier et dans certaines sciences sociales et comportementales. 》.

Afin de mieux comprendre comment un problème philosophique d'ordre moral peut surgir d'une science appliquée comme la médecine et d'ainsi mieux comprendre également la fonction qu'est appelée à remplir la bioéthique, considérons le 
problème de la définition de la mort. Traditionnellement, les philosophes ont défini la mort de plusieurs façons différentes en se fondant sur différentes conceptions ontologiques de la personne humaine (c'est-à-dire en déduisant la première des secondes). Ainsi, on a défini la mort comme "la perte irrémédiable de la conscience », « l'absence de contrôle de soi », « la perte irrémédiable de la raison», "la perte de la capacité d'identité personnelle», «la séparation de l'âme et du corps », etc. De son côté, la profession médicale s'est entendue pendant longtemps pour définir la mort par la cessation des fonctions vitales comme la respiration, la circulation du sang, les battements du coeur, les réflexes, etc. Mais certains développements de la médecine contemporaine allaient sérieusement remettre en question ces définitions : 1 . l'introduction de techniques pour prolonger la vie (comme la respiration artificielle) et 2. le perfectionnement des techniques de transplantation d'organes à partir de cadavres. Il est désormais possible de maintenir artificiellement, à l'aide de machines, la «vie » de patients « morts » au sens de l'ancienne définition médicale, c'est-à-dire dont les fonctions vitales comme la respiration ou la circulation sanguine ont cessé et ne sont plus spontanées et naturelles. Deux issues médicales sont possibles dans cette situation : ou bien les fonctions vitales reprendront leurs activités de façon naturelle et spontanée dans un certain temps indéterminé, ou bien ces activités ne reprendront pas de façon spontanée et naturelle. La première possibilité nous empêche donc de pouvoir appliquer l'ancien critère de la cessation des fonctions vitales.

Le problème de la redéfinition de la mort allait se poser avec d'autant plus d'acuité qu'avec le perfectionnement des techniques de transplantation d'organes, le corps médical allait se trouver face à un problème assez particulier: on sait d'une part qu'une des conditions d'une transplantation réussie d'organes est de pouvoir les retirer du cadavre le plut tôt possible après la mort; d'autre part, il est impératif pour la profession médicale de s'assurer qu'on ne retirera jamais un organe d'une personne encore vivante. Ces problèmes particuliers et très concrets allaient donc forcer le corps médical à redéfinir la mort.

Insistons ici sur la différence entre la définition philosophique de la mort (la déduction à partir de conceptions ontologiques) et sa définition médicale en introduisant une distinction importante 
faite par Roland Pucetti (1982 : 101) entre la "personne biologique " et la " personne morale». Selon Pucetti, l'expression la "personne biologique » ferait référence à l'organisme particulier (membre de l'espèce Homo Sapiens) alors que la "personne morale» ferait référence à la totalité des expériences conscientes d'une personne. Pucetti affirme ensuite, pour bien nous faire comprendre sa distinction, que le temps de vie de la personne morale est nécessairement plus petit (et vice versa) que le temps de vie d'une personne biologique. Il faut compter en effet que la conscience n'apparaît qu'un certain temps t après la naissance et que souvent on constate qu'il y a une dégénérescence de la conscience chez les vieillards qui dure un certain temps $t$ avant la mort. La somme de ces deux temps est la mesure de la différence dans le temps de vie des personnes biologique et morale. Conséquemment, il nous faut donc distinguer entre la mort biologique et la mort morale et se rendre à l'évidence qu'une définition philosophique de la mort morale ne pouvait en aucune façon solutionner les problèmes posés par les développements de la médecine. Non seulement le corps médical avait-il besoin d'une définition de la mort biologique mais encore il avait besoin d'une définition opératoire (cf. Hempel, 1972: 138-42), c'est-à-dire «en spécifiant une opération de vérification bien définie qui lui fournit un critère d'application. $"$. Par exemple, on saura si un liquide est «acide » en y trempant un papier bleu de tournesol et en observant s'il vire au rouge. C'est pourquoi le corps médical optera pour une définition physiologique et neurophysiologique : une personne sera reconnue médicalement morte lorsque 1 . la respiration et les mouvements musculaires spontanés auront cessé et 2 . que l'encéphalogramme indiquera l'absence d'activités cérébrales dans le néocortex. Supposons maintenant que nous ayions défini la mort comme la séparation de l'âme et du corps, il est bien entendu qu'aucune opération à effectuer sur le patient branché sur une machine permettant de le faire respirer artificiellement, n'aurait pu nous permettre de vérifier qu'une telle séparation a eu lieu. Nous dirons que cette dernière définition n'est pas opératoire et qu'en conséquence elle est tout simplement inapplicable dans le cas qui nous intéresse.

Mais plusieurs contesteront qu'il s'agit véritablement d'un problème bioéthique et le considéreront plutôt comme un problème purement médical. Nous n'aurons aucune difficulté à admettre 
cette objection puisque notre but était avant tout de faire voir comment un problème ayant des implications philosophiques importantes (notamment dans le cas de l'euthanasie ou de l'avortement) peut surgir de la pratique médicale et comment, pour être applicable à un problème, une solution doit être opérationnelle. Il est important de noter ici que nous n'avons aucunement l'intention de soutenir que tous les problèmes philosophiques de l'éthique médicale peuvent recevoir des solutions physiologiques ou neurophysiologiques : bien au contraire. Nous voulons plutôt soutenir la thèse selon laquelle même si la solution n'est pas physiologique ou neurophysiologique, elle devra néanmoins être opérationnelle. Une opération (comme observer un comportement ou interroger un agent) devra pouvoir être effectuée qui nous permette de vérifier s'il s'agit d'un cas particulier de la règle morale (comme les opérations médicales permettent de dire que ce cas particulier est bien un cas de mort au sens de la nouvelle définition médicale).

Considérons un autre problème bioéthique, à savoir, celui de l'avortement. Dans les sociétés contemporaines, les femmes ont une multitude de raisons (dont il nous serait impossible de dresser une liste exhaustive) pour demander de se faire avorter, parmi lesquelles mentionnons, à titre d'exemples, les complications cardiaques pouvant mettre leur vie en danger en accouchant, des conditions psychologiques suicidaires ou traumatisantes, la fécondation suite à un viol, les conditions économiques précaires de la famille ou de la fille-mère, etc. Ce sont là des problèmes concrets et pressants à résoudre non seulement pour les femmes mais également pour la société puisque devant une demande accrue, elle se voit forcée de légiférer de façon à restreindre la pratique médicale de l'avortement. Une foule de solutions philosophiques ont été proposées pour résoudre ce problème : par exemple, dans une perspective catholique, si la vie humaine commence dès l'union de l'âme et du corps et que cette union s'opère dès la fécondation alors il en découle que tout avortement est moralement inacceptable et correspond ni plus ni moins à un meurtre. Notons que cette solution est tout à fait opérationnelle au sens défini plus haut: elle interdit une suite de comportements médicaux observables, c'est-à-dire la pratique de l'avortement. Il est donc tout à fait facile à un agent de savoir quand son 
comportement est moralement inacceptable. Notons cependant que contrairement à l'a pproche que nous suggérons ici, cette façon de résoudre le problème s'inscrit dans la perspective traditionnelle qui voudrait fonder l'interdiction de l'avortement (une règle morale) sur des conceptions ontologico-religieuses extra-morales dont le caractère hautement spéculatif rend la possibilité d'un consensus peu probable. Si elle est applicable et « rationnellement » fondée sur des conceptions ontologico-religieuses, il n'en demeure pas moins qu'elle n'est tout simplement pas une solution aux problèmes des femmes et de la société à l'origine de la demande accrue d'avortements. En voulant déduire la solution de conceptions atemporelles ontologiques, la perspective philosophique traditionnelle nie ces problèmes en en faisant complètement abstraction.

Considérons la solution légale apportée à ce problème par la Cour suprême des États-Unis dont on trouvera un extrait du jugement dans T.L. Beauchamp et L. Walters, eds. (1982: 228) :

Nous n'avons nul besoin de résoudre la difficile question du moment où la vie commence. Lorsque ceux spécialisés en médecine, en philosophie et en théologie sont incapables d'en arriver à un consensus, le judiciaire n'est pas, à ce moment du développement de la connaissance humaine, dans une position pour spéculer sur cette question.

Dans l'esprit des juges, il apparaît clairement qu'en se passant complètement de l'approche fondationnelle, on peut néanmoins apporter une solution légale au problème de l'avortement. La solution légale est d'interdire l'avortement après la $28^{\mathrm{e}}$ semaine de grossesse, c'est-à-dire au moment où le fœtus est en mesure de survivre en dehors du ventre de sa mère. Avant ce stade, il revient en définitive à la femme concernée et à son médecin d'opter ou non pour l'avortement. Notons que la solution proposée est opérationnelle au sens précédemment défini et qu'elle représente une solution au problème concret de l'avortement. Ce n'est cependant pas une solution au problème moral de l'avortement car, avant la $28^{\mathrm{e}}$ semaine, la cour ne fait que renvoyer le problème à la femme et au médecin. Si le problème de l'avortement a un aspect social, un aspect médical et un aspect légal, il a également un aspect moral que nous sommes sur le point de cerner plus spécifiquement.

Le fait légal le plus curieux à propos de l'avortement est qu'avant la fin du XIX ${ }^{\mathrm{e}}$ siècle, les lois sur l'avortement aux États- 
Unis étaient beaucoup plus libérales que celles actuellement en vigueur au $\mathrm{XX}^{\mathrm{e}}$ siècle. Cette "dé-libéralisation», dirons-nous, transforme un problème moral en un problème légal. Considérons d'autres exemples pour faire apparaître la distinction entre les deux. Supposons qu'un médecin ayant permis l'intervention d'une sage-femme lors d'un enfantement à l'hôpital soit suspendu par la direction de cet établissement ou encore qu'un médecin soit traduit en cour criminelle pour avoir "débranché » un mourant afin de raccourcir ses souffrances. Il est clair, en de tels cas, que ce qui fera pencher le médecin dans le sens des autorités ne sont pas des raisons morales mais des coercitions externes. Le médecin pourra être moralement en faveur de l'intervention des sages-femmes dans les salles d'accouchement ou de l'euthanasie dans certains cas exceptionnels, mais sera contraint de ne pas agir selon sa propre conscience. Le problème moral devient alors celui de savoir s'il faut obéir à une loi que l'on pense moralement inacceptable dans certains cas. À moins d'avoir une foi absolue en ses principes moraux et d'être prêt à souffrir les pires humiliations, il va de soi qu'on se sentira contraint d'agir malgré nous, d'une certaine façon. Défier les autorités ainsi est un acte de courage que peu de gens seraient prêts à accomplir (en temps normal). Cela exige de vaincre la peur naturelle des représailles (de la punition). Il y a donc ici présent une «force psychologique » naturelle (la peur). Il faut que cela soit bumainement possible de vaincre cette peur et cela n'est pas donné à tous : c'est pourquoi ceux qui iront jusqu'au bout seront considérés comme des «fous» ou des gens très courageux. Peu importe le qualificatif retenu, dans les deux cas, ce seront des déviants par rapport à la moyenne. De même, certains enfants en bas âge pourront faire montre d'un sens moral étonnant mais on ne saurait exiger pour la majorité d'entre eux qu'ils se conduisent moralement en adulte. Sur la base d'une loi de l'évolution appartenant à la psychologie populaire (et confirmée par la psychologie scientifique), nous jugeons bumainement impossible pour la majorité des enfants en bas âge de se comporter moralement comme des adultes. Nous pourrions caractériser ces particularités en disant qu'un comportement acquiert un sens moral, s'il est bumainement possible pour la majorité de l'accomplir et si nous ne sommes pas contraints par des forces externes, rendant bumainement impossible pour la majorité des gens de l'accomplir. 


\section{LA NATURE DES PROBLÈMES DE LA BIOÉTHIQUE}

Au cours de notre analyse de l'aspect synthétique du discours bioéthique, nous avons mis en évidence un autre élément important de la structure épistémologique de son discours, à savoir, les problèmes que la bioéthique est appelée à résoudre. Le trait le plus marquant qui est apparu est que ce sont des problèmes multidisciplinaires. Un problème bioéthique n'a jamais qu'un aspect moral mais également des aspects biologique, médical, légal, psychologique, social, économique, politique, etc. Il ressort clairement de notre analyse que ce sont des problèmes complexes et, du tableau que nous avons dressé de la structure du discours moral, que les moyens théoriques dont dispose l'éthique sont assez limités. L'appareillage conceptuel de l'éthique ne permet de saisir que l'aspect moral des problèmes. Pour simplement comprendre la nature d'un problème bioéthique, il importe d'être au courant de ses différents aspects cernés par un ensemble d'autres disciplines scientifiques. Il apparaît donc tout à fait illusoire de pouvoir trancher dans ces problèmes en se référant uniquement à des règles et des principes moraux. C'est pourtant ce que l'approche philosophique traditionnelle nous conduit à tenter de faire en voulant fonder des règles bioéthiques sur des valeurs suprêmes et des conceptions ontologiques extra-morales et philosophiques.

Mais si, comme nous le préconisons, pour simplement comprendre un problème bioéthique et ainsi pouvoir évaluer ses implications morales d'une façon rationnelle, il nous faut connaître ses autres aspects pertinents, c'est donc dire que les règles morales que nous établirons ou celles que nous chercherons à appliquer devront être compatibles avec les discours de certaines sciences de la nature (comme la biologie ou la médecine) et avec les discours de certaines sciences humaines (comme le droit ou la psychologie). Cette compatibilité est une condition nécessaire pour que le discours bioéthique qui tient compte des multiples aspects de ses problèmes, puisse être logiquement cohérent. Or, dans la mesure où ces autres discours peuvent être rationnellement fondés, leur compatibilité avec le discours moral nous procurera un fondement indirect à la rationalité de ce dernier. Cette façon d'envisager les choses suggère un critère pour évaluer si des règles morales sont plus indirectement rationnelles que d'autres: une règle morale sera d'autant plus indirectement rationnelle qu'elle ne sera pas 
contredite par le discours d'autres disciplines scientifiques pertinentes. Ce fondement rationnel indirect est certainement moins absolu que le fondement sur des conceptions ontologiques que les philosophes traditionnels voulaient conférer à l'éthique. Mais comme ce fondement rationnel direct semble à jamais inatteignable et que le relativisme moral s'est progressivement imposé, il semble plus raisonnable de chercher un autre type de fondement rationnel à l'éthique. Nous pourrions ainsi admettre une certaine forme de relativisme moral sans verser dans le relativisme moral total. Même si les différentes règles morales ne peuvent pas être fondées empiriquement (comme les énoncés descriptifs), ni analytiquement (comme les énoncés mathématiques), ni ontologiquement, certaines règies morales sont néanmoins préférables rationnellement à d'autres du fait qu'elles sont moins contredites par le discours scientifique.

\section{LA VALIDITÉ D'UN DISCOURS BIOÉTHIQUE}

Afin de préciser davantage ce que cela signifie pour un discours bioéthique d'être contredit par le discours d'une science de la nature ou d'une science humaine, considérons à nouveau les cas de la redéfinition médicale de la mort et de l'avortement et comparons-les. La définition médicale de la mort biologique se fonde sur le système conceptuel de la physiologie et de la neurophysiologie: deux sciences de la nature. Une patiente est morte médicalement lorsque ses fonctions vitales (respiration, circulation sanguine, etc.) ont cessé d'agir spontanément et que le néocortex ne fait plus montre d'activités électrico-chimiques. Supposons maintenant que notre ontologie stipule qu'une personne humaine (morale) est plus qu'une simple personne biologique et que ce qui caractérise une personne humaine est sa conscience; nous serons alors conduit par voie de conséquences logiques à définir la «mort» (la fin de la personne humaine) comme étant la perte irrémédiable de la conscience. Notre définition morale de la mort sera compatible avec la définition médicale dans la mesure où nous supposerons que la conscience ne pourrait pas être sans les fonctions vitales et les processus neuronaux. Autrement dit, la vie au sens médical est un présupposé à la vie au sens moral. Si nous croyons cependant qu'un retour de la conscience est toujours possible même trois semaines (disons) après la cessation des 
fonctions vitales spontanées, alors notre définition morale de la mort sera contredite par la définition médicale. En effet, si le corps médical décide qu'après quatre jours seulement de survie artificielle sans récupération des fonctions vitales il est tout à fait légitime de cesser le maintien artificiel de la vie, elle affirme du même coup qu'il est physiquement impossible que la conscience réapparaisse après quatre jours alors que nous soutenons le contraire. Les deux discours sont incompatibles. Selon le critère proposé plus haut la supposition morale selon laquelle la conscience n'est pas possible sans la vie biologique nous semble donc indirectement plus rationnelle (et donc préférable) que celle selon laquelle la conscience est possible même sans la vie biologique. Le cas de la redéfinition de la mort ne fait pas tant problème car les différentes philosophies morales partent de suppositions compatibles avec la définition médicale.

Considérons le cas de l'avortement où la question de la compatibilité est plus problématique. Dans une défense du droit des femmes à l'avortement libre, Judith Jarvis Thomson (1971) avance l'analogie du joueur de violon. Selon cette analogie, vous vous réveillez un de ces matins dans un lit d'hôpital dos à dos avec un violoniste de réputation mondiale ayant perdu conscience. On a découvert qu'il avait une déficience du foie mettant ses jours en péril. C'est pourquoi la Société des mélomanes a décidé d'entreprendre des recherches sur les moyens possibles de guérison. Les membres de cette société ont appris que vous étiez le seul capable de le guérir car vous êtes d'un type sanguin compatible et c'est pourquoi ils ont décidé de vous kidnapper et de brancher le système sanguin du violoniste sur le vôtre afin que votre foie purifie son sang en même temps que le vôtre. Le directeur de l'hôpital qui attendait votre réveil s'approche de vous et d'un air désolé exprime sa désapprobation du comportement des membres de la Société des mélomanes mais il vous avertit également qu'il ne pourra vous débrancher du système sanguin du violoniste avant neuf mois ou peut-être même neuf ans (ou même toute votre vie). Débrancher les deux systèmes sanguins équivaudrait à tuer le violoniste. J.J. Thomson reconstruit alors sur cette base l'argument fondamental de ceux qui sont contre l'avortement même lorsque la vie de la mère est en danger et même lorsque la grossesse survient à la suite d'un viol. Le violoniste (comme le zygote, l'embryon ou le 
forus) est un être humain ; or, tous les êtres humains ont droit à la vie; donc le violoniste a droit à la vie. Bien sûr vous avez également le droit de décider de votre vie et de contrôler votre propre corps, mais ces droits sont hiérarchiquement inférieurs au droit d'une personne à la vie. J.J. Thomson est convaincue que la majorité des personnes (homme ou femme) qui se retrouveraient dans une telle situation trouveraient tout à fait outrageux de ne pas pouvoir décider elles-mêmes de l'utilisation de leur propre corps et du déroulement de leur vie et donc de ne pas pouvoir décider elles-mêmes de se faire «avorter » du violoniste. La raison en est que nous n'avons pas consenti librement à une telle situation et que nous n'avons donc pas à nous sentir responsable de la mort du violoniste.

Comme le remarque avec justesse Mary Anne Warren (1973: 253):

Cet argument est de prime abord très plausible et dans le cas extrême de grossesse à la suite d'un viol, il est probablement concluant.

Mais, il comporte néanmoins une limitation importante : le viol est le seul cas où la situation de la femme est véritablement analogue à celle du violoniste, c'est-à-dire qu'elle n'est aucunement responsable de ce qui lui arrive. Pour la majorité des cas normaux de grossesse non-désirée cependant, on ne pourrait pas la dégager de toute responsabilité : elle aurait pu rester chaste, elle aurait pu prendre ses pilules plus méthodiquement, elle aurait pu utiliser d'autres méthodes contraceptives, etc. L'analogie ne tient donc pas pour la majorité des cas normaux. Néanmoins, c'est un argument de poids pour le cas exceptionnel mentionné plus haut. En ce cas, la seule règle morale qu'on pourrait invoquer est la parabole du bon samaritain : la personne qui accepte de se dévouer pour un pur étranger malgré qu'elle ne soit aucunement responsable de ses difficultés. Mais les «bons samaritains » ne courent pas les rues comme s'empresse de le faire remarquer J.J. Thomson (1971: 60) :

[...] ceci apparaît peut-être plus manifestement dans l'histoire de Kitty Genovese, qui, comme vous vous souviendrez, a été assassinée devant trente-huit personnes pouvant la voir ou l'entendre sans qu'aucune ne lui vienne en aide. Un bon samaritain se serait précipité pour l'aider à se défendre contre le meurtrier (nous soulignons). [...] Mais les trentehuit personnes non seulement n'ont pas fait cela mais aucune n'a même pris la peine d'appeler la police au téléphone. 
Considérons ce jugement moral de plus près : nous avons

1. une règle morale: la parabole du bon samaritain

2. une situation typique : aider quelqu'un qui est agressé par une tierce personne

3. des jugements moraux :

3.1. ne pas aider Kitty Genovese est moralement condamnable

3.2. ne pas laisser vivre le violoniste est moralement condamnable

3.3. ne pas laisser vivre un fœtus est moralement condamnable (même si dans ces trois cas, personne n'est responsable)

4. des situations particulières: les comportements et les circonstances entourant:

4.1. le cas de Kitty Genovese

4.2. l'analogie du célèbre violoniste

4.3. le cas d'une grossesse indésirée en cas de viol.

Ce jugement moral comporte manifestement les mêmes carences que nous reprochions à l'approche traditionnelle, à savoir, de vouloir trancher unilatéralement sur la base du seul système conceptuel de l'éthique, une question impliquant un ensemble d'autres aspects du ressort d'autres disciplines. Demandons-nous, pour notre part, si ce jugement moral et cette règle morale sont compatibles avec le discours scientifique. Et notamment le discours de la psychologie sociale qui à partir de Darley et Latané (1968) s'est intéressé de façon toute particulière au cas de Kitty Genovese. L'énoncé existentiel singulier décrivant le fait est le même en bioéthique comme en psychologie. Il y a donc bien un aspect synthétique et des énoncés descriptifs dans le discours moral puisque, sur la description des faits, les deux disciplines se rejoignent. En 1964, dans la ville de New York une jeune fille s'est fait poignarder par un meurtrier et malgré qu'elle se soit défendue énergiquement et qu'elle ait crié pendant presque une demi-heure, aucun des trente-huit témoins qui l'ont vue ou entendue n'est intervenu ou n'a appelé la police. Le discours moral tentera de montrer que ce comportement particulier (des trente-huit témoins) dans ces circonstances particulières peut être subsumé sous la situation typique décrite par la parabole du bon samaritain. Il s'agit ensuite d'évaluer la situation particulière : puisque le comportement 
des trente huit témoins n'est pas conforme au comportement du bon samaritain, il est donc condamnable moralement sur cette base. La parabole et cette évaluation suppose, comme nous le faisions remarquer à la fin de la section précédente, qu'il est humainement possible d'adopter le comportement du bon samaritain et que cela ne dépend que de la volonté de chacun.

À partir du même fait, le psychologue cherchera pourquoi les trente-huit témoins ont agi de cette façon. Pour expliquer ce phénomène social, Darley et Latané avancent une hypothèse, à savoir, celle de la diffusion de la responsabilité dans des situations sociales. Il s'agit toujours de subsumer ce cas particulier sous un cas typique mais plutôt que d'évaluer le comportement, on cherchera à le comprendre. Selon cette hypothèse, dans une situation sociale de ce genre, plus il y a de témoins qui sont conscients de la présence des autres témoins et plus la possibilité qu'ils interviennent activement diminue. Pour tester empiriquement cette hypothèse, les psychologues imaginent une expérimentation où une personne réclame de l'aide par interphone à trois autres personnes qui ne peuvent se voir mutuellement: à une des personnes on laisse entendre qu'elle est seule sur l'interphone avec celle réclamant de l'aide; la seconde croit qu'il y a deux autres personnes sur l'interphone et la troisième qu'il y en a cinq. Les résultats sont suffisamment significatifs : dans $85 \%$ des cas où la personne se croit seule, elle se porte rapidement au secours de la personne en détresse; dans $62 \%$ des cas où l'on croit que seulement deux personnes sont impliquées et dans $31 \%$ des cas où l'on croit cinq personnes impliquées, on fera la même chose. Autrement dit, les trente-huit témoins ne sont pas intervenus pour aider Kitty Genovese non pas parce qu'ils ne voulaient pas venir au secours d'une personne en détresse, mais bien parce qu'ils étaient conscients qu'un grand nombre d'autres personnes pouvaient observer le meurtre et entendre la victime crier : ils ont décidé en conséquence de laisser les autres intervenir. Le phénomène a été surnommé l'«effet du témoin». Notons que cette découverte va à l'encontre du sens commun pour lequel il est logique de croire que plus il y a de témoins dans une situation d'urgence et plus il est probable qu'au moins un des témoins interviendra. On ne saurait donc tenir les trente-huit témoins responsables de ne pas être au courant de cet effet du témoin. Dans ces conditions, les personnes impliquées ont 
réagi selon une tendance normale et aucun n'aurait pu être au courant d'une hypothèse qui sera formulée quatre ans plus tard.

Il ressort clairement de cette analyse que la règle morale et le jugement moral sont contredits par le discours psychologique au niveau de leur supposition fondamentale, à savoir, que dans cette situation, il était humainement possible pour la majorité de se comporter comme le bon samaritain. L'effet du témoin montre, au contraire, qu'il était humainement impossible pourla majorité des gens de réagir de la façon recommandée par la règle morale dans cette situation. Le concept de responsabilité du discours moral est incompatible avec celui du psychologue social dans la mesure où la responsabilité au sens moral n'est pas quelque chose qui peut être « diffusée ». Si les témoins ont véritablement été victimes de l'effet du témoin, alors on ne saurait moralement les blâmer pour des actions dont ils ne sont pas véritablement responsables. Ceci ne signifie pas qu'aucune règle morale sanctionnant l'aide que nous devons apporter aux autres personnes en détresse n'est possible. Cependant, une règle tenant compte du fait qu'il y a peu ou beaucoup de témoins serait indirectement plus rationnelle (donc préférable) que la parabole du bon samaritain. En conséquence, cette règle serait hypothétique et non pas catégorique. Notons que le bon samaritain était le seul à pouvoir intervenir dans cette situation et qu'il n'y a donc pas d'effet de diffusion.

Quelles conséquences pouvons-nous tirer de ces discussions en ce qui concerne le problème de l'avortement? Premièrement, on ne saurait assimiler le cas d'une grossesse indésirée au cas de Kitty Genovese, même en cas de viol, car la femme et le médecin sont les seuls témoins à pouvoir intervenir dans la situation et ils en sont conscients. C'est donc une situation où l'effet du témoin ou le phénomène de la diffusion de la responsabilité en situation sociale d'urgence est à peu près inexistant. Il semble donc de prime abord que l'adoption du comportement du bon samaritain soit humainement possible dans le cas des grossesses indésirées à la suite de viol et donc qu'elle puisse être moralement sanctionnée. Mais ce serait considérablement réduire la portée de notre concept de compatibilité. Nous ne visons pas la compatibilité du discours moral uniquement avec celui de la psychologie mais également avec celui de l'ensemble des autres sciences de la nature et humaines. Deuxièmement donc, il est illusoire de penser qu'on 
pourrait trancher une question aussi complexe que celle de la moralité de l'avortement simplement en déduisant la règle morale l'interdisant de conceptions ontologiques extra-morales sur la personne humaine ou sur la vie ou encore en montrant qu'une telle déduction est logiquement impossible. Troisièmement, une telle solution est superfétatoire dans la mesure où si la règle morale ainsi déduite n'est pas compatible avec l'ensemble des sciences de la nature et humaines, le comportement qu'elle recommande sera humainement impossible à performer et donc la règle ne sera pas applicable. Si, par contre, les conceptions ontologiques extramorales permettent de déduire une règle morale qui est compatible avec les discours scientifiques alors il y a lieu de se douter que le discours moral et le discours scientifique ont la même ontologie même si leurs formulations respectives sont différentes. Il en découle quatrièmement que la compatibilité (ou l'incompatibilité) d'une règle morale guidant nos décisions dans la pratique de l'avortement avec le discours scientifique est un fondement rationnellement plus adéquat que la compatibilité (ou l'incompatibilité) avec des conceptions ontologiques atemporelles et purement a priori. Autrement dit, on ne saurait proposer une règle morale sur l'avortement sans également prendre en considération ses aspects sociologique, psychologique, économique, politique, etc. Cinquièmement, cette règle morale, en admettant des exceptions lorsque l'adoption du comportement n'est pas humainement possible, ne pourrait être catégorique mais simplement hypothétique. Ainsi, des conditions psycho-socio-économiques particulières rendant la grossesse ou la naissance d'un enfant humainement impossible pourraient empêcher la subsomption d'un cas particulier sous cette règle. C'est pourquoi nous sommes conduits à conclure qu'une règle morale interdisant catégoriquement toute forme d'avortement peu importent les circonstances est tout simplement indirectement moins rationnelle (donc inacceptable) qu'une autre permettant des exceptions. L'absence totale de toute règle morale à cet effet n'est pas moins indirectement irrationnelle que son interdiction catégorique. Pour reprendre l'exemple de M.A. Warren ( $1973: 255)$, une femme qui changerait d'idée sur le fait d'avoir un enfant et se ferait avorter parce que cela lui empêcherait de faire un voyage de plaisance en Martinique suppose une femme à ce point frivole et inconsciente qu'il est humainement impossible (au sens de très exceptionnelle) de réaliser un tel comportement. Une 
telle conception ontologique de la femme est non seulement incompatible avec les données des sciences humaines mais également avec le sens commun. Notons que la question de savoir qui devrait prendre la décision morale est un autre problème qui est probablement plus du ressort de la politique et de la justice que de la bioéthique.

\section{CONCLUSION}

En conclusion, il importe de faire le point brièvement sur ce que nous avons dit jusqu'ici. Nous sommes partis d'un constat d'échec en ce qui concerne le fondement rationnel (pour ceux dont c'était le but évidemment) que la philosophie morale traditionnelle et la métaéthique voudraient conférer à l'éthique et à la bioéthique. Bien au contraire, ces entreprises fondationnelles contribuent (par voie de conséquence) à l'établissement de plus en plus ferme du relativisme moral. Or, le relativisme total en matière de morale nous apparaissant hautement indésirable (ne serait-ce que pour ses conséquences sur les rapports sociaux auxquels nous n'avons d'autre choix que de participer), il nous importait de trouver un autre fondement rationnel à l'éthique susceptible non pas d'invalider le relativisme moral mais du moins d'en limiter l'expansion.

Même en admettant le relativisme moral, croyons-nous, il est possible de choisir d'une façon rationnellement justifiée entre deux règles morales et d'en trouver une préférable. Pour ce faire cependant, il nous a fallu renverser complètement les perspectives de la philosophie morale traditionnelle (la déduction à partir de conceptions ontologiques extra-morales) et de la métaéthique (l'insistance sur la caractérisation négative de l'éthique ayant pour point de référence absolu la science.)

On pourrait tenter de caractériser d'une façon générale en termes kantiens le renversement proposé en adoptant l'impératif catégorique exactement opposé à celui de Kant, à savoir, «De ne jamais faire d'une maxime guidant notre action une loi strictement universelle». Mais cette caractérisation impliquant une autocontradiction (un impératif nous enjoignant de ne jamais adopter un impératif catégorique est lui-même un impératif catégorique), nous proposons plutôt de changer de perspective et d'abandonner l'idée de fonder rationnellement des règles éthiques et bioéthiques 
en en faisant des lois universelles ou des principes suprêmes et inconditionnels. Il vaudrait mieux sur ces questions retrouver la sagesse aristotélicienne en matière juridique. Selon Aristote, en effet, une loi (juridique), en raison même de son caractère universel, ne peut prévoir tous les cas particuliers et a donc toujours besoin de correctifs ou d'équité. Il n'en va pas autrement, à notre avis, en éthique et en bioéthique pour les raisons suivantes que nous formulerons sous forme de thèses:

T1: Les discours éthique et bioéthique disposent de moyens théoriques trop limités n'offrant la possibilité de saisir qu'un aspect (moral) des problèmes multidisciplinaires qu'elles entendent résoudre et, en conséquence, une solution simplement moralement fondée ne sera jamais suffisante.

T2: Vouloir fonder les règles morales sur des conceptions ontologiques extra-morales revient à nier le caractère multidisciplinaire de ces problèmes et conduit à proposer des solutions humainement inapplicables.

T3: La compatibilité (ou l'incompatibilité) des règles morales avec le discours des sciences de la nature et des sciences humaines est en mesure de nous permettre de déterminer ce qui est humainement réalisable et ce qui ne l'est pas en regard de l'adoption de certains comportements dans des situations typiques.

T4: Si l'éthique et la bioéthique doivent être un savoir pratique, leur compatibilité (ou leur incompatibilité) avec le discours des sciences de la nature et celui des sciences humaines est un fondement rationnel indirect préférable épistémologiquement au fondement direct conféré par leur compatibilité avec des conceptions ontologiques atemporelles et a priori.

T5: La conséquence est qu'une règle (ou un principe) moral sera toujours hypothétique et jamais catégorique.

T6: Contrairement à la supposition fondamentale du droit, nous ne sommes pas tous égaux (ex aequo) devant les règles morales. Par exemple, le psychologue ayant connaissance de l'effet du témoin dans le cas de Kitty Genovese 
serait moralement plus responsable que le commun des mortels se fiant au sens commun.

T7 : Une règle morale compatible avec le discours scientifique nous apparaît préférable épistémologiquement (du point de vue d'un choix rationnellement justifié) à une règle incompatible avec ce savoir.

L'avantage de la fondation rationnelle indirecte que nous proposons est d'admettre le relativisme moral tout en bloquant le relativisme total. Selon notre thèse, nous pouvons être rationnellement justifiés de préférer certaines règles morales à d'autres. Le changement de perspective proposé cependant n'a pas pour but d'invalider complètement tout le savoir éthique accumulé par la philosophie morale traditionnelle. La hiérarchie des valeurs et des conceptions ontologiques a certainement mis en évidence des aspects importants des problèmes moraux. De même, il ne saurait être question de contredire C. Panaccio (1987) qui insiste sur la contribution importante de la métaéthique à l'éthique. Tout en conservant le plus de connaissances éthiques possibles dans ces traditions, nous recommandons de changer la perspective ou la façon habituelle de considérer l'éthique et la bioéthique.

Notre perspective repose sur un ensemble de suppositions dont nous voudrions discuter en terminant. Pour fonder nos décisions morales, nous avons le choix entre deux perspectives. Ceci ne veut pas dire qu'il n'y en a pas d'autres possibles mais, dans l'état actuel de nos connaissances sur ces questions, ce sont les deux qui s'offrent à nous. La première, la perspective traditionnelle, s'intéresse à des débats très spéculatifs sur des énoncés de très haut niveau de généralité et d'abstraction. Sa tâche consiste à montrer que de certaines conceptions ontologiques, théologiques, philosophiques ou populaires on peut déduire la priorité de certains principes moraux sur d'autres. Nos conduites morales devraient tendre à éviter toute incompatibilité avec ces conceptions a priori devant être acceptées comme des dogmes universels et éternels. Ainsi, dans la perspective catholique, un fidèle qui place ses croyances religieuses au-dessus de la loi ne devrait pas hésiter à défier une injonction (quitte à faire de la prison) pour manifester son opposition à l'avortement. Par exemple, si par un malheureux 
hasard électoral, un parti politique prônant l'extermination systématique des personnes âgées ou des infirmes venait à prendre le contrôle de l'appareil judiciaire et politique, il ne fait aucun doute qu'un bon nombre de citoyens et citoyennes défieraient la loi. De même, un médecin sensible à l'inutilité de faire endurer de longues souffrances à un patient dont la seule issue est la mort, serait tenu moralement d'accomplir un comportement contraire à la loi interdisant l'euthanasie. Étant donné les poursuites judiciaires qui s'ensuivront, ie médecin doit faire face à un dilemme moral déchirant car ses principes et ses idéaux ainsi bafoués, il sera conduit à démissionner devant sa tâche morale. En effet, si le médecin obéit à la loi en étant moralement en désaccord avec elle, il devient un simple exécutant reléguant ses responsabilités morales à une autorité extérieure à lui. La majorité des gens impliqués dans de telles situations, sentant que cela est au-dessus de leurs forces que de s'opposer à l'appareil judiciaire, se résigneront en se blâmant moralement ou en étant blâmés par d'autres. De notre point de vue, ce résultat est le signe manifeste de l'irréalisme de la perspective traditionnelle en éthique. La situation à laquelle fait face le médecin n'est pas que morale (comme dans une discussion philosophique sur la mort de Socrate, par exemple) mais également légaie, sociale, psychologique, politique, etc. La simple compatibilité avec des conceptions abstraites peu importent les circonstances réelles équivaut ni plus ni moins qu'à une forme de platonisme (le monde réel n'étant qu'une apparence d'un monde idéal) et à une solution insatisfaisante pour les problèmes qui nous intéressent. En effet, puisque les principes moraux dont il est question sont des conséquences de certaines conceptions extra-morales spéculatives, nous sommes conduits à devoir débattre des questions éthiques à de hauts niveaux d'abstraction et à en faire des débats philosophicoontologiques complètement détachés de la réalité quotidienne.

Plutôt que de planer dans les hautes sphères de la spéculation philosophique, la perspective que nous proposons voudrait ramener les débats éthiques "sur la Terre» en posant que tout principe moral est bypotbétique (jamais catégorique). Autrement dit, aucun principe moral n'a de valeur en soi (ou peu importent les circonstances réelles) mais uniquement en regard d'une situation réelle et d'autres principes moraux compétitifs. Il apparaît plus réaliste et rationnel de dire que dans certaines situations réelles, certains 
principes moraux sont préférables à d'autres et que dans l'évaluation de ces situations réelles, un ensemble de disciplines scientifiques ont également leur mot à dire, à savoir, de déterminer ce qu'il est bumainement possible pour la majorité de faire dans une situation réelle. En d'autres mots, une règle morale nous commandant d'accomplir un comportement au-dessus de nos forces et de celles de la majorité des êtres humains, nous apparaît irréaliste et de ce fait rationnellement injustifiable - par exemple, exiger d'un enfant qu'il se comporte moralement comme un adulte.

La compatibilité avec le discours des sciences de la nature et des sciences humaines nous apparaît un meilleur critère que la déduction à partir de conceptions ontologico-spéculatives pour décider de la valeur d'une règle morale dans une situation réelle. Mais il est clair que la rationalité de l'éthique sera ainsi indirectement fondée si le discours scientifique peut être lui-même rationnellement fondé et que ses résultats soient considérés comme étant valides. C'est là une présupposition qui est loin d'être évidente - il nous faut bien le reconnaître - si nous cherchons un fondement absolu. Mais, là n'est pas notre intention. C'est par rapport aux conceptions ontologico-théologico-philosophico-populaires qu'il nous faut apprécier la rationalité et la validité de la connaissance scientifique. Sur ce plan, la science apparaît préférable du fait que ses conceptions sont contrôlées sur le plan expérimental alors que les conceptions spéculatives sont des idéaux dont le réalisme n'est pas justifié.

On rétorquera certainement que, bien souvent, comme dans le cas de l'effet du témoin, les données expérimentales sont statistiques et de ce fait, invalides. Ainsi, si dans $69 \%$ des cas, on n'agit pas en bon samaritain, il reste $31 \%$ des cas où l'on agit de la façon recommandée par la parabole. Il est donc bumainement possible d'adopter le comportement recommandé. On peut même soutenir que les témoins ne réagissant pas « manquent de perspective morale » et que les autres ( $39 \%$ ) sont, au contraire, foncièrement moraux. Deux problèmes sont ici en cause: un concernant la nature du savoir psychologique et un autre concernant l'universalité d'une éthique. La psychologie est une science théorique visant à identifier des « forces » et des mécanismes psychologiques à l'œuvre dans certaines situations réelles. Parmi ces « forces», la volonté de se conformer à des principes moraux en est certainement une mais ce n'est pas la seule. Il ne fait aucun doute qu'une situation 
expérimentale en psychologie puisse être interprétée en termes moraux mais cela équivaut à réduire l'être humain à n'être mû que par une seule force dominante, à savoir, la force morale. Clairement c'est là une simplification excessive d'un être humain réel, une idéalisation qui demande à être tempérée par la psychologie ou la sociologie. Si on peut toujours affirmer que les personnes ayant réagi ont agi moralement (quoique des motifs inconscients égoïstes puissent toujours être considérés), on ne peut pas affirmer aussi clairement que les personnes n'ayant pas agi sont moralement condamnables ou manquent de perspective morale. Préalablement à l'action morale, il faut évaluer correctement la situation. Si les témoins n'avaient pas conscience de la gravité de la situation et des dangers que courraient Kitty Genovese (dont nous-mêmes nous avons pris conscience seulement après l'événement et en n'y étant pas impliqués), on ne peut certes les blâmer moralement. Tout comme l'enfant est déterminé par le stade de son évolution auquel il se trouve, les témoins sont victimes (disons) de la dépersonnalisation des rapports sociaux dans les grandes villes...

Considérons le problème de l'universalité d'une éthique. Pour pouvoir remplir leur fonction, des règles morales ne peuvent exiger d'accomplir des comportements au-dessus des forces de la majorité des personnes. On ne saurait nier que l'expression «humainement possible» est plutôt floue mais étant donné la teneur des discussions en cause, il n'est nul besoin d'avoir une précision mathématique dans sa définition. Est-ce possible, par exemple, de composer des pièces musicales à six ou sept ans? Strictement parlant, en pensant à Mozart, par exemple, nous devons répondre oui. De toute évidence cependant cela n'est pas bumainement possible pour la majorité des enfants de cet âge sinon Mozart ne serait pas un enfant prodige. On ne saurait donc exiger de ceux qui apprennent la musique à cet âge qu'ils composent des symphonies et ensuite les blâmer s'ils ne réussissent pas. Il n'en va pas autrement de l'éthique. À moins de prôner une éthique pour une petite élite, il va de soi qu'une éthique réaliste s'adresse à la majorité. Pour être réaliste et rationnel, une règle morale doit recommander un comportement moral qu'il est humainement possible à la majorité d'accomplir dans une situation normale. Que cette impossibilité ne soit pas strictement universelle et admette des exceptions trouve sa meilleure expression dans des données 
statistiques. Pour que l'effet du témoin soit strictement universel, il faudrait qu'il n'y ait aucun autre facteur impliqué dans cette situation et cela, non seulement l'hypothèse des psychologues ne l'affirme pas mais encore, notre perspective ne l'implique pas. Bien au contraire, nous avançons l'idée que ce sont des situations pluridisciplinaires où de multiples facteurs (autres que purement moraux ou purement psychologiques) sont impliqués. Nous n'affirmons pas que seul l'effet du témoin devrait être considéré mais uniquement qu'il devrait également être considéré.

Peut-être la principale difficulté que doit affronter notre perspective est qu'elle semble présupposer une distinction nette entre les énoncés scientifiques et les énoncés éthiques. Si la valeur d'une éthique doit être appréciée selon sa compatibilité avec le discours scientifique, ne doit-on pas supposer que ce dernier discours est exempt de toute considération éthique? Cette supposition nous apparaît irréaliste du fait que même l'entreprise scientifique est soumise à des règles éthiques (l'honnêteté intellectuelle, par exemple). Mais, comme nous le disions, nous ne cherchons pas un fondement absolu. Cette interdépendance entre éthique et science montre simplement le caractère multidisciplinaire de ces problèmes qu'il faut prendre en considération.

Mais pourquoi ne pas tout simplement abandonner l'entreprise même de fondation en éthique et en bioéthique? Pourquoi vouloir fonder rationnellement des règles morales? En fait, ce n'est pas une éthique ou des règles morales que nous cherchons à fonder rationnellement mais bien l'application de certaines règles morales dans certaines situations. Comme cette application donne lieu à des désaccords manifestes, la discussion rationnelle même très critique nous semble hautement préférable à l'expression catégorique de nos croyances personnelles (la foi) ou au recours à la force, à la violence et à la désobéissance civile.

Faculté de philosophie

Université Laval 


\section{RÉFÉRENCES BIBLIOGRAPHIQUES}

BEAUCHAMP, T.L. (1982), «Ethical Theory and Bioethics» dans Beauchamp et Waters (eds.) : $1-43$.

BEAUCHAMP, T.L. et WALTERS, L. (eds.) (1982), Contemporary Issues in Bioetbics. Second Edition, Belmont, California, Wedsworth Publishing Co.

COUTURE, Jocelyne (1986), «Méta-éthique» dans Cabiers d'épistémologie, No. 8609, Montréal, Université du Québec L'article paraîtra dans le premier volume ("Problématiques») de l'Encyclopédie philosophique, A. Jacob (Dir.), Paris, Presses Universitaires de France.

DARLEY, J.M. et LATANE, B. (1968), « Bystander Intervention in Emergencies. Diffusion of Responsability " dans Journal of Personality and Social Psychology, $8: 377-83$.

GAUTHIER, D. (1986), Morals by Agreement, Oxford, Clarendon Press.

GEWIRTH, A. (1982), Human Rights. Essays on Justification and Applications, Chicago, University of Chicago Press.

HARSANYI,J.C. (1982), "Morality and the Theory of Rational Behavior » dans A. Sen et B. Williams (eds.), Utilitarianism and Beyond, Cambridge, University Press: 37-63.

(1976), "Advances in Understanding Rational Behavior" dans J.C. Harsanyi, Essays on Ethics, Social Bebavior and Scientific Explanation, Dordrecht/Boston, D. Reidel : 89-117.

HEMPEL, C.G. (1972), Éléments d'épistémologie, Trad. B. Saint-Servin, Paris, Armand Colin (Collection «U2 »).

HUDSON, W.D. (ed.) (1969), The Is-Ought Question. A Collection of Papers on the Central Problem in Moral Pbilosopby, New York, St-Martin Press.

(1970), Modern Moral Philosophy, New York, Doubleday Publishers.

HUME, David (1971), Abrégé du Traité de la nature bumaine, Trad. D. Deleule, Paris, Aubier Montaigne. Press.

(1978), A Treatise of Human Nature, Oxford/New York, Clarendon

KAUFMANN, J.N. (1987a), «Être et Devoir » dans Cabiers d'épistémologie, No. 8701, Montréal, Université du Québec. Paraîtra dans la section "Problématiques» de l'Encyclopédie Philosophique, Vol. 1, Dir. A. Jacob, Paris, Presses Universitaires de France.

(1987b), "Suffit-il d'agir rationnellement pour agir moralement?" dans Dialogue, XXVI, No. 4 : 715-29.

KRIPKE, S. (1982), «Troisième conférence » dans La logique des noms propres, Trad. P. Jacob et F. Récanati, Paris, Les éditions de Minuit: 95-144.

MOORE, G.E. (1903), Principia Ethica, Cambridge, University Press. (1912), Ethics, London, House University Library. 
PANACCIO, Claude (1987), "L'analyse logique du discours moral ", dans Actes du Colloque: L'éthique à venir: une question de sagesse? Rimouski, Université du Québec, Éditions du Groupe de Recherche ETHOS : 291-8.

PERELMAN, C. (1980), Introduction historique à la philosopbie morale, Bruxelles, Éditions de l'Université de Bruxelles.

POPPER, K.R. (1959), The Logic of Scientific Discovery, New York/Evanston, Harper and Row.

PUCCETTI, R. (1982), «The Life of a Person» dans Bondeson, W.B. et autres (eds.), Abortion and the Status of the Foetus, Dordrecht/Holland, D. Reidel Publishing Co. Repris dans Beauchamps, T.L. et Walters L. (eds.) : 101-7.

QUINE, W.V.O. (1953) “Two Dogmas of Empiricism », dans From a Logical Point of View, New York, Harper And Row, 1961 : 20-46.

SEARLE, J.R. (1969), Speech Acts, Cambridge, University Press, Chap. : 8

THOMSON, J.J. (1971), “A Defense of Abortion» dans Pbilosophy and Public Affairs, Vol. 1, No. 1 : 47-66.

WARREN, M.A. (1973), “On the Moral and Legal States of Abortion » dans The Monist, Vol. 57, No. 1, repris dans Beauchamps et Walters (eds.) : 250-60. 